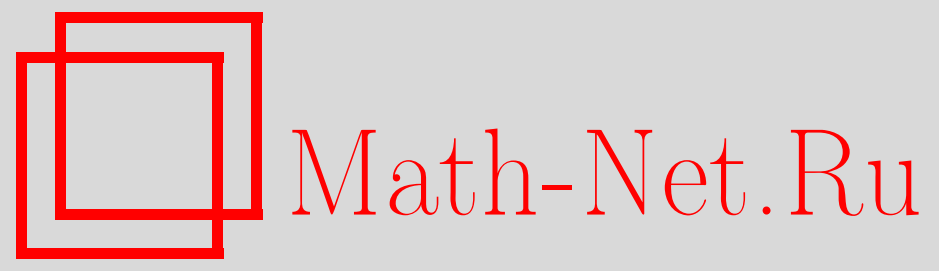

И. Г. Шапошников, О некоторых системах образующих симметрической и знакопеременной групп, допускающих простую программную реализацию, Дискрет. матем., 2004, том 16, выпуск 1, 114-120

DOI: https://doi.org/10.4213/dm146

Использование Общероссийского математического портала Math-Net.Ru подразумевает, что вы прочитали и согласны с пользовательским соглашением http://www . mathnet.ru/rus/agreement

Параметры загрузки:

IP : 54.166 .219 .16

26 апреля 2023 г., 14:27:51

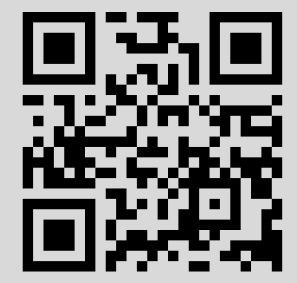


УдК 512.6

\title{
О некоторых системах образующих симметрической и знакопеременной групп, допускающих простую программную реализацию
}

() 2004 г. И. Г. Шапошников

\begin{abstract}
Приводятся системы образующих симметрической и знакопеременной групп подстановок, при программной реализации которых используются простые арифметические операции. Для некоторых предложенных систем образующих дается оценка глубины групп (индекса системы образующих).
\end{abstract}

\section{1. Введение}

В [1] была поставлена задача построения систем образующих симметрических групा, допускающих простую программную реализацию и достаточно быстро порождающих симметрическую группу. Быстрота порождения характеризуется числовыми параметрами, связанными с числом элементов системы образующих в произведениях, через которые выражаются элементы симметрической группы. Напомним в этой связи некоторые определения из [1].

Каждый элемент $g$ конечной группы $G=\langle M\rangle$, порожденной множеством элементов $M$, представляется в виде произведения элементов из $M$. Минимальное число $l$ сомножителей среди таких представлений элемента $g$ называется длиной элемента $g$ группы $G$ в системе образующих $M$. Максимальная длина элемента группы $G$ в системе образующих $M$ называется длиной групшы $G$ в системе образующих $M$.

Условимся подмножества из группы $G$ умножать как комплексы: $A \cdot B$ есть множество всех элементов вида $a \cdot b$, где $a \in A, b \in B$. Множество $M^{i}$ называется $i$-м слоем группы $G$ в системе образующих $M$. Минимальное $s$ такое, что $\left|M^{s}\right|=\left|M^{s+1}\right|$, называется глубиной группы $G$ в системе образующих $M$ и обозначается $\partial(G ; M)$. В англоязычной литературе вместо термина глубина используется термин индекс системы $M$.

Если $G$ есть группа подстановок некоторого конечного множества $\Omega$, то показатель $k$-транзитивности множества $M$ есть минимальное натуральное $l$, для которого множество подстановок $M^{l}$ является $k$-транзитивным, если такое $l$ существует, в противном случае этот показатель полагается равным $\infty$. Данный параметр обозначается $\partial_{k}(M)$.

В представленной работе описываются некоторые системы образующих симметрических и знакопеременных групп, допускающих простую программную реализацию. Так как вычислительные устройства обрабатывают двоичные числа, мы будем прежде всего 
рассматривать подгрупшы симметрической группы $S\left(V_{N}\right)$ подстановок множества $N$-разрядных двоичных чисел $V_{N}$. Простыми программно реализуемыми операциями являются операцин сложения по модулю 2 и модулю $2^{N}$, операции умножения по модулю $2^{N}$, операции обращения в память и некоторые логические операции. При обращении в некоторую область памяти реализуются унарные отображения вида $V_{k} \rightarrow V_{N}$.

\section{2. Системы образующих симметрической и знакопеременных групп, построенных с помощью операций колец вычетов}

Рассмотрим системы образующих

$$
M_{a, c}(m, r, \varphi)=\left\{f_{c, d}, g_{a, b}: d \in Z_{m-r}, b \in Z_{m}\right\}, \quad c \in Z_{m-r}, \quad a \in Z_{m},
$$

включающие подстановки $g_{a, b}$ и $f_{c, d}$ на множестве вычетов $Z_{m}$ по модулю $m: g_{a, b}(x)=$ $a x+b(\bmod m)$ и $f_{c, d}(\varphi(x))=\varphi(c x+d(\bmod m-r))$, если $x<m-r$ и $f_{c, d}(\varphi(x))=\varphi(x)$, если $x \geqslant m-r$. Здесь $(a, m)=1,(c, m-r)=1,0<r<m-1, \varphi-$ биективное отображение $Z_{m}$ на себя.

Предложение 1. Показатель 2-транзитивности множества $M_{a, c}(m, 1, \varphi)$ не больше mpex.

Доказательство. Зафиксируем пары элементов $(A, B),(C, D)$ множества $Z_{m}, A \neq B$, $C \neq D$. Выберем такие подстановки $h_{1}, h_{2}$ из множества $G_{a}=\left\{g_{a, b}: b \in Z_{m}\right\}$, для которых $h_{1}(A)=\alpha, h_{2}(\alpha)=C$, где $\alpha=\varphi(m-1)$. Это можно сделать, так как множество подстановок $G_{a}$ транзитивно. Пусть $h_{2}^{-1}(D)=\beta, h_{1}(B)=\gamma$. Так как множество подстановок $\left\{f_{c, d}: d \in Z_{m-1}\right\}$ транзитивно на $Z_{m} \backslash \varphi(m-1)$, можно выбрать подстановку $f_{c, d}$ такую, что $f_{c, d}(\gamma)=\beta$. Нетрудно видеть, что подстановка $h_{2} f_{c, d} h_{1}$ переводит $(A, B)$ в $(C, D)$. Утверждение доказано.

Предложение 2. Группа, порожденная $M_{a, c}(m, r, \varphi),(r, m)=1$, дважды транзитивна.

Доказательство. Заметим, что группа $G$, порожденная $M_{a, c}(m, r, \varphi)$, примитивна. Действительно, элементы множества $X=\{\varphi(x): m>x \geqslant m-r\}$ должны целиком составлять блоки импримитивности. Однако из условия $(r, m)=1$ такие блоки могут состоять только из одного элемента. Так как $G_{X}$ транзитивна на $Z_{m} \backslash X$, по теореме Жордана [2] группа $G$ дважды транзитивна.

Предложение 3. Пусть $\varphi(x)=x+i(\bmod m)$ для $x \in Z_{m-r}$, тогда группа, порожденная $M_{a, c}(m, r, \varphi)$, включает в себя знакопеременную группу на $Z_{m}$.

Доказательство. Через $T(i, j)$ при $j>i$ будем обозначать цикл вида $(i, i+1, \ldots, j)$, а при $j=i$ - тождественную подстановку. Так как элемент $a$ обратим в кольце вычетов $Z_{m}$, для для $b$ и $e$, удовлетворяющих соотношению $b-e=a(\bmod m)$, справедливо равенство $g_{a, e}^{-1} g_{a, b}=T(0, m-1)$. Заметим, что $T(0, m-1)^{-i} f_{c, d} T(0, m-1)^{i}(x)=c x+d$ $(\bmod m-r)$ для $x<m-r$ и $T(0, m-1)^{-i} f_{c, d} T(0, m-1)^{i}(x)=x$ для $x \geqslant m-r$. Так как элемент $c$ обратим в кольце вычетов $Z_{m-r}$, для $d$ и $l$, удовлетворяющих соотношению $d-l=c(\bmod m-r)$, справедливо равенство

$$
T(0, m-1)^{-i} f_{c, l}^{-1} f_{c, d} T(0, m-1)^{i}(x)=T(0, m-r-1) .
$$


Отсюда нетрудно видеть, что группа $G$, порожденная системой образующих $M_{a, c}(m, r, \varphi)$, примитивна. Так как $G$ содержит тройной цикл

$$
(0, m-r, 1)=T(0, m-1) T(0, m-r-1) T(0, m-1)^{-1} T(0, m-r-1)^{-1},
$$

по теореме Жордана [2] она включает знакопеременную группу. Утверждение доказано.

Системы образующих $M_{a, c}(m, r, \varphi)$ в ряде случаев допускают простую программную реализацию. Например, рассмотрим отображение $\varphi_{0}(i)=i$ при $i$, не равных $0, m-1$, и $\varphi_{0}(0)=m-1, \varphi_{0}(m-1)=0$. При $m=2^{N}, r=1$ системы образующих $M_{1,1}\left(2^{N}, 1, \varphi_{0}\right)$ реализуются с помощью простых арифметических операций над $N$-разрядными двоичными числами: $f_{1, d}(0)=0 ; f_{1, d}(x)=x+d$, если $x+d<2^{N}$ и $x \neq 0 ; f_{1, d}(x)=x+d+1$ $\left(\bmod 2^{N}\right)$, если $x+d \geqslant 2^{N}$ и $x \neq 0$.

Предложение 4. В системе образующих $M_{1,1}\left(m, 1, \varphi_{0}\right)$ глубина симметрической группы не превосходит $2 m-3$.

Доказательство. Заметим, что множества $\left\{f_{1, d}: d \in Z_{m-1}\right\}$ и $\left\{g_{1, b}: b \in Z_{m}\right\}$ элементов системы образующих $M_{1,1}\left(m, 1, \varphi_{0}\right)$ составляют циклические групшы $F$ и $G$, порожденные соответственно $T(1, m-1)$ и $T(0, m-1)$.

При $0 \leqslant i<j \leqslant m-1$ справедливо соотношение

$$
T(0, m-1)^{i} T(1, m-1)^{j-i} T(0, m-1)^{-j}=T(i, j) .
$$

Действительно,

$$
T(0, m-1)^{-j}(x)=x-j \quad(\bmod m) ;
$$

если $m>x>j$ или $0 \leqslant x<i$, то

$$
T(0, m-1)^{i} T(1, m-1)^{j-i}(x-j)=x ;
$$

если $i \leqslant x<j$, то

$$
T(0, m-1)^{i} T(1, m-1)^{j-i}(x-j)=x+1 ;
$$

наконец, если $x=j$, то

$$
T(0, m-1)^{i} T(1, m-1)^{j-i}(0)=i .
$$

Любую подстановку $h: a_{i} \rightarrow i, i=0,1, \ldots, m-1$, можно получить с помощью произведения циклов $T(i, j)$ :

$$
h=T\left(m-2, b_{m-2}\right) \ldots T\left(1, b_{1}\right) T\left(0, a_{0}\right),
$$

где $b_{i}=T\left(i-1, b_{i-1}\right) \ldots T\left(1, b_{1}\right) T\left(0, a_{0}\right)\left(a_{i}\right)$.

По предложению 1 переходы $a_{0} \rightarrow 0$ и $a_{1} \rightarrow 1$ можно обеспечить подстановкой вида $T(0, m-1)^{x} T(1, m-1)^{y} T(0, m-1)^{z}$. Поэтому

$$
h=T\left(m-2, b_{m-2}\right) \ldots T\left(2, b_{2}\right) T(0, m-1)^{x} T(1, m-1)^{y} T(0, m-1)^{z},
$$

где $b_{i}=T\left(i-1, b_{i-1}\right) \ldots T\left(2, b_{2}\right) T(0, m-1)^{x} T(1, m-1)^{y} T(0, m-1)^{z}\left(a_{i}\right)$.

Действительно, подстановки $T\left(j, b_{j}\right)$ при $j>i$ оставляют на месте элемент $i$ :

$$
T\left(i, b_{i}\right) \ldots T\left(2, b_{2}\right) T(0, m-1)^{x} T(1, m-1)^{y} T(0, m-1)^{z}\left(a_{i}\right)=i .
$$

Таким образом, любая подстановка $h$ симметрической группы $S\left(Z_{m}\right)$ присутствует в множестве $(G F)^{l} G$ при $l=m-2$. Утверждение доказано. 


\section{3. Система образующих знакопеременной группы, построенной с помощью операций сложения по модулю 2 и модулю $2^{N}$}

Обозначим через $\Sigma_{N}$ регулярное представление групшы $V_{N}(\oplus)$, где $\oplus$ - покоординатное сложение $N$-разрядных двоичных векторов по модулю 2.

Через $\sigma_{x}$ будем обозначать подстановки множества $V_{N}$, определяемые операцией сложения с элементами $x \in V_{N}$ по модулю 2: $\sigma_{x}(i)=i \oplus x$.

Множество элементов $\left\{f_{1, d}\right\}$ системы образующих $M_{1,1}\left(2^{N}, 1, \varphi_{0}\right)$ будем обозначать через $F$. Нетрудно видеть, что $F$ - циклическая группа, порожденная элементом $f=f_{1,1}$. Как было замечено в предыдущем разделе, элементы группы $F$ легко реализуются с помощью операция сложения по модулю $2^{N}$.

Предложение 5. Глубина знакопеременной группы в системе образующих $\left\{\Sigma_{N}, F\right\}$ не превосходит $N 2^{N+2}-2^{N+2}+4 N+C$, где $C$ - челое число, не зависящее от $N$.

Доказательство. Так как $\Sigma_{N}$ и $F$-группы четных подстановок, то $\left\langle\Sigma_{N}, F\right\rangle$ - подгруппа знакопеременной групшы. Легко проверить, что

$\sigma_{2} f=(2,1,0) \cdot\left(3,6,5,4,7, \ldots, 4 i+2,4 i-1,4 i, 4 i+3, \ldots, 2^{N}-2,2^{N}-3,2^{N}-4,2^{N}-1\right)$.

Следовательно, в примитивной группе $\left\langle\Sigma_{N}, F\right\rangle$ лежит цикл длины 3 , поэтому по теореме Жордана она совпадает со знакопеременной [2].

Нетрудно показать, что при $i=0,1, \ldots, N-2$

$$
\begin{aligned}
f^{2^{i+1}} \sigma_{2^{i}} f^{-2^{i+1}} \sigma_{2^{i}} & =\left(0,2^{i+1}\right)\left(2^{i}, 2^{i}+2^{i+1}\right)=h_{i+1}, \\
f \sigma_{2^{N-1}} f^{-1} \sigma_{2^{N-1}} & =(0,1)\left(2^{N-1}, 2^{N-1}+1\right)=h_{N} .
\end{aligned}
$$

Кроме того, $h_{0}=\left(h_{1} h_{N}\right)^{2}=(0,1)(2,3)$.

Заметим, что при $x=i-2^{j-1}, i>0, i+2^{j} \leqslant 2^{N}-1, j \geqslant 1$ подстановка

$$
f^{x} h_{j} f^{-x}=\left(0, i+2^{j-1}\right)\left(i, i+2^{j}\right)
$$

переводит $i$ в $i+2^{j}$ и оставляет на месте элементы $i+1+2^{j}, \ldots, 2^{N}-1$, а подстановка

$$
f^{x} h_{0} f^{-x}=(0, i-1)(i, i+1), \quad i \geqslant 2,
$$

при $x=i-2, i+1 \leqslant 2^{N}-1$, переводит $i$ в $i+1$ и оставляет на месте элементы $i+2, \ldots, 2^{N}-1$.

Для любых двух элементов $a, b \in V_{N}$, удовлетворяющих условиям $a<b, b>3$, построим подстановку в алфавите $\left\{\Sigma_{N}, F\right\}$, переводящую $a$ в $b$ и оставляющую на месте любой элемент $c>b$.

Рассмотрим следующие случаи.

Начнем с пассмотрения случая $a \neq 0$. Для того, чтобы перевести $a$ в $b$ и оставить на месте любой элемент $c>b$, необходимо перемножить подстановки вида (1) и (2) в количестве, равном числу единиц в двоичном представлении числа $\boldsymbol{b}-\boldsymbol{a}$.

В случае, когда $a=0, b=2^{j}, j \geqslant 2, a$ в $b$ переводит подстановка

$$
f^{2^{j-2}} h_{j-1} f^{-2^{j-2}} h_{j-1}=\left(0,2^{j}, 2^{j}-1,2^{j-1}+2^{j-2}, 2^{j-2}\right),
$$


оставляя на месте любой элемент $c>b$.

В случае, когда $a=0, b \neq 2^{j}$, для того, чтобы перевести $a$ в $b$ и оставить на месте любой элемент $c>b$, достаточно сначала перевести 0 в 4 подстановкой $f h_{1} f^{-1} h_{1}=(0,4,2,3,1)$, а затем умножить эту подстановку на произведение подстановок вида (1) и (2) в количестве, равном числу единиц в двоичном представлении числа $b-4$.

Используя полученные результаты, построим в алфавите $\left\{\Sigma_{N}, F\right\}$ подстановку $h$ знакопеременной групшы, переводящую элементы $a_{2}, a_{3}, \ldots, a_{2^{N}-1}$ соответственно в $2,3, \ldots, 2^{N}-1$, полагая

$$
h=v(2,3) \mu\left(b_{4}, 4\right) \ldots \mu\left(b_{2^{N}-3}, 2^{N}-3\right) v\left(2^{N}-2,2^{N}-1\right),
$$

где $v\left(2^{N}-2,2^{N}-1\right)$ - подстановка из множества $\left(\Sigma_{N} F \Sigma_{N}\right)$, переводящая $a_{2^{N}-2}$ в $2^{N}-2$ и $a_{2^{N}-1}$ в $2^{N}-1$ (по предложению 1$) ; \mu\left(b_{i}, i\right)$ - подстановка, переводящая

$$
b_{i}=\mu\left(b_{i+1}, i+1\right) \ldots \mu\left(b_{2^{N}-3}, 2^{N}-3\right) v\left(2^{N}-2,2^{N}-1\right)\left(a_{i}\right)
$$

в $i$ и оставляющая на месте $i+1, \ldots, 2^{N}-1 ; v(2,3)$ - подстановка, переводящая

$$
b_{i}=\mu\left(b_{4}, 4\right) \ldots \mu\left(b_{2^{N}-3}, 2^{N}-3\right) v\left(2^{N}-2,2^{N}-1\right)\left(a_{i}\right)
$$

в $i, i=2,3$, и оставляющая на месте $4,5, \ldots, 2^{N}-1$.

Согласно выше приведенным рассуждениям,

$$
\mu\left(b_{i}, i\right) \in\left(F \Sigma_{N} F \Sigma_{N} F\right)^{r_{i}-1}\left(\Sigma_{N} F\right)^{4},
$$

где $r_{i}$ - максимальное число единиц в двоичном представлении числа, не превосходящего $i$. Далее, заметим, что подстановки $h_{0}, h_{1}$ и $f h_{1} f^{-1} h_{1}$ порождают знакопеременную группу на множестве $\{0,1,2,3,4\}$. Отсюда следует, что подстановка $v(2,3)$ лежит в множестве $\left(F \Sigma_{N}\right)^{B}, B$ - некоторая постоянная.

Окончательно получаем, что подстановка $h$ содержится в множестве $\left(\Sigma_{N} F\right)^{l} \Sigma_{N}$ при $l=2 R+2\left(2^{N}-6\right)+C_{1}$, где $C_{1}-$ некоторое целое число,

$$
R=\sum_{i=4}^{2^{N}-3} r_{i}
$$

Пусть $k_{i}$ - максимальное число, удовлетворяющее условию $2^{k_{i}}-1 \leqslant i$. Тогда $r_{i}=k_{i}$, a

$$
R=\sum_{j=2}^{N-1} j 2^{j}-(N-1)-2=N 2^{N}-2^{N+1}+N-1 .
$$

Следовательно, любая подстановка знакопеременной групшы лежит в множестве $\left(\Sigma_{N} F\right)^{l} \Sigma_{N}, N>2$, при $l=N 2^{N+1}-2^{N+1}+2 N+C$. Утверждение доказано.

\section{4. Метод использования сплетения универсальных алгебр для построения систем образующих симметрической группы}

Пусть $\delta$ - отображение множества $B^{n}$ в множество всех отображений $A^{n}$ на $A ; \delta$-сплетением [4] алгебры $(B ; \omega)$ с операцией $\omega: B^{n} \rightarrow B$ называется алгебра $\left(A \times B ; \omega^{(\delta)}\right)$ с 
операцией $\omega^{(\delta)}:(A \times B)^{n} \rightarrow A \times B$, определяемой соотношением

$$
\omega^{(\delta)}\left(\left(a_{1}, b_{1}\right), \ldots,\left(a_{n}, b_{n}\right)\right)=\left(\delta\left(b_{1}, \ldots, b_{n}\right)\left(a_{1}, \ldots, a_{n}\right), \omega\left(b_{1}, \ldots, b_{n}\right)\right) .
$$

Алгебра $\left(A \times B ; \omega^{(\delta)}\right)$ несет информацию о $(B ; \omega)$, поэтому, задавая требуемые свойства алгебры $(B ; \omega)$ и строя ее $\delta$-сплетения, мы можем ожидать получения заданных свойств уже у алгебр с большей мощностью основных множеств. Так как алгебра $\left(A \times B ; \omega^{(\delta)}\right)$ является гомоморфным прообразом алгебры $(B ; \omega)$ [4], данный метод обобщает известные методы использования гомоморфных прообразов, например, метод построения ортогональных латинских квадратов [5], использование скрещенного произведения квазигрупп [6] и сплетения $n$-квазигрупп [7]. В [8] были приведены примеры использования метода $\delta$-сплетения универсальных алгебр для построения преобразований с заданными свойствами. Приведем пример применения метода $\delta$-сплетения для построения систем образующих симметрических групп, допускающих простую программную реализацию. Рассмотрим систему образующих подстановок симметрической группы $S\left(V_{N}\right)$ вида $\Gamma_{N} h$, где $\Gamma_{N}$ - регулярное представление группы $V_{N}(+),+-$ операция сложения по модулю $2^{N}, h$ - некоторая подстановка. Вопросы порождения симметрической группы данной системой образующих исследовались в [1]. При реализации данной системы образующих возникает задача выбора просто реализуемой подстановки $h$. Для решения данной задачи воспользуемся конструкцией сплетения.

Пусть даны $h_{0} \in S\left(V_{k}\right), N>k$, отображение $\delta: V_{k} \rightarrow S\left(V_{N-k}\right)$. Определим $\delta$-сплетение подстановки $h_{0}$, полагая $h_{0}^{(\delta)}(a, b)=\left(h_{0}(a), \delta(a)(b)\right)$, где $a \in V_{k}$ - старшие, а $b \in V_{N-k}$ - младшие разряды двоичного числа $(a, b)$. В ряде случаев подстановка $h_{0}^{(\delta)}$ допускает простую программную реализацию. Например, рассмотрим отображение $h$ вида $h(a, b)=(a, b) \oplus \chi(a)$, где $a \in V_{k}, b \in V_{N-k}, \chi: V_{k} \rightarrow V_{N}$ - унарное отображение, реализуемое обращением к таблище, состоящей из $2^{k} \quad N$-разрядных двоичных векторов. Нетрудно видеть, что данное отображение $h$ будет подстановкой, если отображение $\chi$ имеет вид $\chi(a)=\left(h_{0}(a) \oplus a, \alpha_{a}\right)$, где $h_{0}$ - подстановка на множестве $V_{k}, \alpha_{a} \in V_{N-k}$. В указанном случае подстановка $h$ представляет собой $\mu$-сплетение подстановки $h_{0}$, где $\mu$ - отображение $V_{k}$ в группу $\Sigma_{N-k}$ :

$$
h(a, b)=h_{0}^{(\mu)}(a, b)=\left(h_{0}(a), b \oplus \alpha_{a}\right) .
$$

Заметим, что при $k=1$ такого типа подстановки исследовались в [1].

Предложение 6. Если группа $\left\langle\Gamma_{k}, h_{0}\right\rangle$ 2-транзитивна и существуют $a, c \in V_{k}$ такие, что подстановка $\delta(a)$ не меняет четности числа, а $\delta(c)$, наоборот, меняет, то группа $\left\langle\Gamma_{k}, h_{0}^{(\delta)}\right\rangle$ примитивна.

Доказательство. Докажем утверждение от противного. Пусть группа $\left\langle\Gamma_{k}, h_{0}\right\rangle$ импримитивна, тогда четные и нечетные числа лежат в разных блоках импримитивности. Пусть $\left(a_{1}, b_{1}\right),\left(a_{2}, b_{2}\right) \in V_{N}$ лежат в одном блоке импримитивности, $a_{1}, a_{2} \in V_{k}, b_{1}, b_{2} \in V_{N-k}$. По условию предложения существуют значения $x_{1}, x_{2}, \ldots, x_{l} \in V_{k}$ такие, что

$$
\begin{aligned}
& \left.x_{l}+h_{0}\left(x_{l-1}+\ldots+h_{0}\left(x_{1}+a_{1}\right) \ldots\right)\right)=a, \\
& \left.x_{l}+h_{0}\left(x_{l-1}+\ldots+h_{0}\left(x_{1}+a_{2}\right) \ldots\right)\right)=c .
\end{aligned}
$$

Заметим, что

$$
\begin{aligned}
& \left(x_{l}, 0\right)+h_{0}^{(\delta)}\left(\left(x_{l-1}, 0\right)+\ldots+h_{0}^{(\delta)}\left(\left(x_{1}, 0\right)+\left(a_{1}, b_{1}\right) \ldots\right)\right)=\left(a, d_{1}\right), \\
& \left(x_{l}, 0\right)+h_{0}^{(\delta)}\left(\left(x_{l-1}, 0\right)+\ldots+h_{0}^{(\delta)}\left(\left(x_{1}, 0\right)+\left(a_{2}, b_{2}\right) \ldots\right)\right)=\left(c, d_{2}\right),
\end{aligned}
$$


причем числа $\left(a, d_{1}\right),\left(c, d_{2}\right)$ по предположению лежат в одном блоке импримитивности, то есть или оба четны, или оба нечетны. Тогда и числа $h_{0}^{(\delta)}\left(a, d_{1}\right), h_{0}^{(\delta)}\left(c, d_{2}\right)$ должны лежать в одном блоке импримитивности, однако, как нетрудно видеть последнее не выполняется по условию предложения. Получаем противоречие, доказывающее утверждение.

Из результатов работы [3] следует, что в условиях предложения 6 группа $\left\langle\Gamma_{N}, h_{0}^{(\delta)}\right\rangle$ является симметрической, если не совпадает с $P G L(2, p)$, где $p=2^{N}-1$ - простое число. Последнее выполняется, если, например, число $2^{N}-1$ не простое или порядок групшы $\left\langle\Gamma_{k}, h_{0}\right\rangle$ больше $|P G L(2, p)|=2^{N}\left(2^{N}-1\right)\left(2^{N}-2\right)$.

\section{Список литературы}

1. Глухов М. М., Задание конечных групп системами образующих. Труды по дискретной математике (1997) 1, 43-66.

2. Wielandt H., Finite permutation groups. Academic Press, London, 1964.

3. Погорелов Б. А., Примитивные группы подстановок, содержащие $2^{m}$-цикл. Алгебра и логика (1980) 19, №2, 236-247.

4. Шапошников И. Г., О конгруэнциях конечных многоосновных универсальных алгебр. Дискретная математика (1999) 11, №3, 48-62.

5. Raghavarao D., Constructions and combinatorial problems in design of experiments. Wiley, New York, 1971.

6. Белоусов В. Д., Основы теории квазигрупп и луп. Наука, Москва, 1967.

7. Борисенко В. В., Неприводимые $n$-квазигруппы на конечных множествах составного порядка. Матем. исследования (1979), №51, 36-42.

8. Шапошников И. Г., Метод использования $\delta$-сплетения универсальных алгебр для построения преобразований с заданными свойствами. Обозрение прикладной и промышленной математики (2002) 9, №3, 669-670.

Статья поступила 23.09.2003. 